Check for updates

Cite this: Mater. Adv., 2020, 1,658

Received 25th March 2020, Accepted 1st June 2020

DOI: $10.1039 / \mathrm{d} 0 \mathrm{ma} 00133 \mathrm{c}$

rsc.li/materials-advances

\section{Novel cost-effective acceptor:P3HT based organic solar cells exhibiting the highest ever reported industrial readiness factor $\dagger$}

\author{
Thomas Rieks Andersen, (D)*a Anne Therese Weyhe, ${ }^{b}$ Qiang Tao, ${ }^{\text {bc }}$ Feng Zhao, ${ }^{a}$ \\ Ran Qin, (D) a Shuhua Zhang, ${ }^{a}$ Hongzheng Chen (D) *a and Donghong Yu (D) *bd
}

\begin{abstract}
Solution-based organic solar cells (OSCs) offer great potential prospects within low-cost electricity production. High-performance materials are traditionally low bandgap polymers requiring a multitude of synthetic steps, limiting the scalability of the materials. A very scalable polymer is poly(3-hexylthiophene) (P3HT) which has recently reached a power conversion efficiency (PCE) of $6.4 \%$ with a non-fullerene acceptor (NFA) O-IDTBR, herein we present four novel simplified NFA analogues to O-IDTBR for reducing the synthetic complexity. The best performing material combination reached an average efficiency of $5.39 \%$, which led to an industrial readiness factor (i-FOM) of 0.26 which is more than a $30 \%$ increase compared to that for P3HT:O-IDTBR. Due to the promise of this material combination, upscaling to large area/ITO-free slot-die coating was conducted reaching an optimized PCE of $3.58 \%$.
\end{abstract}

\section{Introduction}

For Organic solar cells (OSCs) to become economically viable, an important element to consider would be the production cost of the end product, i.e. electricity. ${ }^{1-4}$ This is mainly influenced by the cost of manufacture, power conversion efficiency (PCE), stability of the produced OSCs, and installation \& maintenance (which will not be further evaluated, herein). ${ }^{2}$ The cost of manufacture has two main inputs: materials and processing. It is well-known that processing has to be conducted using techniques which are roll-to-roll compatible, as highthroughput is a necessity for reducing the processing cost, such techniques include slot-die coating, flexographic printing, screen printing, and to some extent thin-film vacuum deposition methods. ${ }^{5-9}$ In contrast, the cost of materials is a more debatable subject as a trade-off between cost of materials and the PCE of the produced OSCs are generally present. As record performances have only been achieved with highly complex molecules requiring a multitude of synthetic steps and limiting

\footnotetext{
${ }^{a}$ State Key Laboratory of Silicon Materials, Department of Polymer Science and Engineering, Zhejiang University, Hangzhou 310027, China. E-mail:0619091@zju.edu.cn,.hzchen@zju.edu.cn

${ }^{b}$ Department of Chemistry and Biosciences, Aalborg University, Aalborg East, DK-9220, Denmark. E-mail: yu@bio.aau.dk

${ }^{c}$ Hunan Provincial Key Laboratory of Environmental Catalysis \& Waste Recycling, School of Chemistry and Chemical Engineering, Hunan Institute of Engineering, Xiangtan, China

${ }^{d}$ Sino-Danish Center for Education and Research, Aarhus, DK-8000, Denmark

$\dagger$ Electronic supplementary information (ESI) available. See DOI: 10.1039/d0ma00133c
}

the overall yield, thereby increasing the material costs. ${ }^{10-13}$ Whereas P3HT is generally considered as the ideal donor (D) candidate for upscaling of OSCs with its simple synthesis, high hole mobility and high stability towards photo-oxidation. However, OSCs prepared with such simple low-cost materials as P3HT:PCBM commonly exhibit performance limitations, due to reduced photon absorption and large energy loss. These performance limitations have been overcome/improved by the implementation of non-fullerene acceptors (NFA) ${ }^{14,15}$ for devices paired with P3HT with $V_{\mathrm{OC}}$ as high as $1.22 \mathrm{~V}^{16}$ and the extended absorption from $\sim 650 \mathrm{~nm}$ to $\sim 850 \mathrm{~nm}$, leading to PCEs surpassing $6 \%$ when O-IDTBR ${ }^{17}$ and P3HT:SF(DPPB $)_{4}{ }^{18-21}$ were chosen. While somewhat simplified electron acceptors such as DFPCBR still reaches efficiencies of $5.25 \% .{ }^{22}$ The highest efficiency achieved for P3HT based devices were reported recently by $\mathrm{Xu}$ et al. therein they presented an impressive device efficiency of up to $8.25 \%,{ }^{23}$ however, this efficiency was only achieved through time-dependent ink modifications which definitely reduces the scalability of the ink system. Therefore, P3HT:O-IDTBR is still viewed as the most likely candidate for upscaling, even though the material system achieved an impressive PCE, later studies of the industrial figure of merit or industrial readiness (i-FOM) revealed that this material combination only scored 0.17 whereas 0.7 has been hypothesized as the required value. ${ }^{11}$ The i-FOM score is deducted from the relationship among device efficiency, stability, and synthetic complexity, revealing that simpler molecules with a reduced synthetic complexity could reduce the gap to the required i-FOM.

Herein we present two approaches for simplifying O-IDTBR to improve the obtained i-FOM value. Initially the benzothiadiazole 
was omitted to a avoid large number of synthetic steps, thereby reducing the synthetic complexity, moreover the electron donating core was substituted with less fused moieties to either adopt costeffective starting materials or reduce their synthetic complexity ${ }^{24}$ even further and also to evaluate the effect of a core degree of fusing. The molecules presented herein are 4,9-dihydro-4,4,9,9tetraoctyl-s-indaceno[1,2- $\left.b: 5,6-b^{\prime}\right]$-dithiophene-dicarbaldehyde, 6-(5-formylthiophen-2-yl)-4,4-dioctyl- $4 H$-indeno[1,2- $b]$ thiophene2-carbaldehyde, 5,5,10,10-tetraoctyl-5,10-dihydrothieno[ $\left[2^{\prime}, 3^{\prime}: 3,4\right]$ cyclopenta[1,2-g]thieno[3,2-c]isochromene-2,8-dicarbaldehyde and 5,5'-(2,5-bis((2-ethylhexyl)oxy)-1,4-phenylene)bis(thiophene-2-carbaldehyde) flanked by 3-ethylrhodanine. The novel acceptors were initially characterized through NMR, MALDI-TOF, UV-Vis absorption and cyclic voltammetry. Their photovoltaic properties were firstly tested and optimized on small area spin-coated OSCs with P3HT as the donor polymer, after which the most promising material was transferred to large-area ITO-free slot-die coated OSCs.

\section{Results}

\section{Physical properties}

The chemical structure of the four novel electron acceptors (A1-A4) can be seen in Fig. 1A. The core molecules were synthesized according to literature ${ }^{25-28}$ after which Knoevenagel condensation between 3-ethylrhodanine and the dialdehyde functionalized core molecules were utilized to obtain the designed electron acceptors with a yield of $71.6 \%, 50.0 \%, 37.8 \%$, and $60.1 \%$ for A1, A2, A3, and A4, respectively, further synthetic details can be seen in supporting information as well as molecular characterization (NMR Fig. S7 and MS Fig. S8, ESI $\dagger$ ). The planarity of the prepared molecules was investigated through density functional theory (DFT) as seen in Fig. 1B, A1 was calculated to be essentially planar whereas both A2 and A3 appears less planar due to their non-fused core, e.g., for A2, the non-fused half of the molecule has a steric twist disrupting planarity and conjugation. The introduction of the ether bond into the fused part of the core molecule seems to create an additional steric twist within A3, resulting in a slight twisting of the fused part of the molecule. The calculations for $\mathbf{A 4}$ also displayed a dominantly planar molecule with just the slightest steric twisting. The increased planarity of $\mathbf{A 1}$ promotes an increase in conjugation length which in turn manifested in a red-shift of the absorption onset of $33 \mathrm{~nm}$ when compared to A2 (Fig. 1C and Table 1). Moreover, A1 also displays an enhanced J-aggregation strength (a relative enhancement in the primary absorption peak (first peak/ plateau from the right in the absorption spectra) compared to the secondary absorption peak) which is associated with a reduced
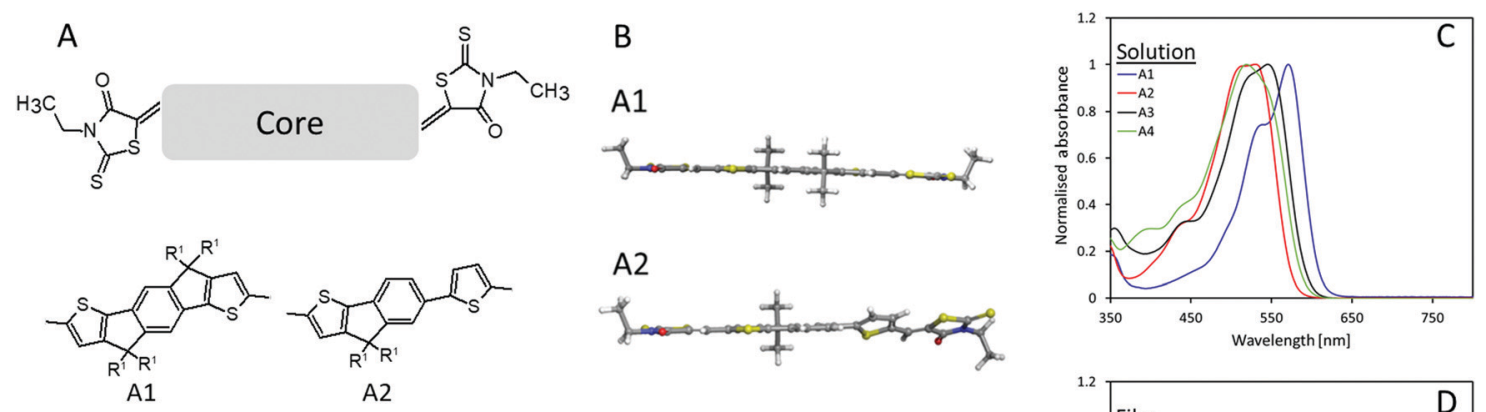

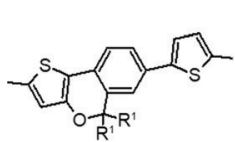

A3

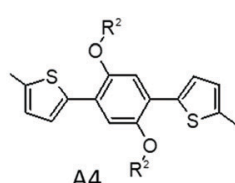

$\mathrm{R}^{1}=$ octyl

$\mathrm{R}^{2}=$ 2-ethylhexyl
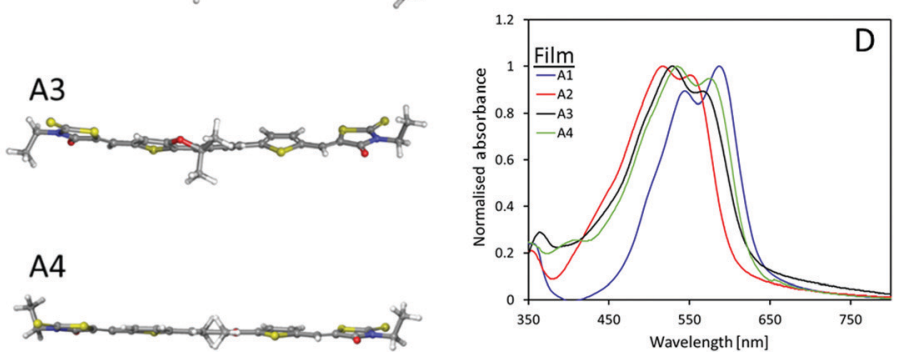

Fig. 1 (A) Chemical structures of $\mathbf{A} 1, \mathbf{A} 2, \mathbf{A} 3$, and $\mathbf{A} 4$; (B) optimized conformation of A1-A4 as calculated by DFT (B3LYP/6-31G*) with methyl groups replacing alkyl chains for clarity; (C) absorbance spectra of A1-A4 in chloroform solution; (D) absorbance spectra of A1-A4 films cast from chloroform solution.

Table 1 Optical and electrical characteristics of $\mathbf{A} 1, \mathbf{A} 2, \mathbf{A} 3$, and $\mathbf{A} 4$, namely absorption onset and maximum absorption for both $\mathrm{CHCl}_{3}$ solution and film, optical band gap $\left(E_{\mathrm{g}}^{\mathrm{opt}}\right)$, cyclic voltammetry determined band gap $\left(E_{\mathrm{g}}^{\mathrm{EC}}\right)$ and $\mathrm{HOMO}$ and LUMO levels positioning

\begin{tabular}{|c|c|c|c|c|c|c|c|c|}
\hline & $\begin{array}{l}\text { Absorbance onset } \\
\text { solution [nm] }\end{array}$ & $\begin{array}{l}\lambda_{\mathrm{Max}} \text { solution } \\
{[\mathrm{nm}]}\end{array}$ & $\begin{array}{l}\text { Absorbance } \\
\text { onset film [nm] }\end{array}$ & $\begin{array}{l}\lambda_{\operatorname{Max}} \\
\text { film [nm] }\end{array}$ & $\begin{array}{l}E_{\mathrm{g}}^{\mathrm{opt} a} \\
\text { film }[\mathrm{eV}]\end{array}$ & $E_{\mathrm{g}}^{\mathrm{EC} b}[\mathrm{eV}]$ & $\begin{array}{l}\text { HOMO } \\
\operatorname{level}^{c}[\mathrm{eV}]\end{array}$ & $\begin{array}{l}\text { LUMO } \\
\text { level }^{d}[\mathrm{eV}]\end{array}$ \\
\hline A1 & 608 & 1.74 & 637 & 587 & 1.95 & 2.06 & -5.61 & -3.55 \\
\hline $\mathbf{A 4}$ & 587 & 2.14 & 629 & 535 & 1.97 & 2.14 & -5.71 & -3.57 \\
\hline
\end{tabular}


voltage loss. ${ }^{29-31}$ The film absorption (Fig. 1D) for all four materials exhibit red-shifts in their onset in a range of 29-42 $\mathrm{nm}$, however, whereas A1 and A4 presents a $\lambda_{\text {Max }}$ red-shift of $16 \mathrm{~nm}, \mathbf{A 2}$ and A3 have slight blue-shifts in $\lambda_{\text {Max }}$ of 13 and $17 \mathrm{~nm}$, respectively, which implies a hinderance in $\pi-\pi$ stacking likely due to the steric twists within the molecules. ${ }^{32,33}$ The absorbance spectra of blended films in a P3HT: acceptor of 1:1 can be found in Fig. S1 ESI. $\dagger$

Cyclic voltammetry (CV) (ESI, $\dagger$ Fig. S2) of the thin films shows that A1-A3 have very similar highest occupied molecular orbital (HOMO) values around $-5.6 \mathrm{eV}$ whereas $\mathbf{A 4}$ possess a slightly lower one with $-5.7 \mathrm{eV}$. As for the lowest unoccupied molecular orbital (LUMO), A1, A3, and A4 present very similar values in the range of $-3.55 \mathrm{eV}$ to $-3.59 \mathrm{eV}$ while $\mathbf{A} 2$ displaying a slightly higher one of $-3.41 \mathrm{eV}$. The LUMO level of P3HT was measured as comparison to be $-2.74 \mathrm{eV}$ which would allow for sufficient energetic offset for optimum energy transfer between the donor and the acceptor(s). These acceptors are therefore all suitable for the preparation of OSCs pairing with P3HT as a donor.

\section{Photovoltaic properties}

Solar cells were prepared with P3HT as the donor and A1-A4 as the acceptor, respectively, due to their matching energy levels
(Fig. 2A) and low synthetic complexity. Devices were prepared in a normal geometry with a device stack of glass/ITO/PEDOT:PSS Al4083/Active layer/ZnO/Ag as displayed in Fig. 2B, similar to previously reported. ${ }^{34}$ All material combinations were optimized for annealing conditions (see ESI $\dagger$ ), D:A ratio (Fig. 3A, C, E and G), and active layer film thickness (Fig. 3B, D, F and $\mathrm{H}$ ), the specific photovoltaic characteristics can be found in ESI, $\dagger$ Fig. S3-S6 for A1-A4, respectively. The optimized $J-V$ curve for the four material combinations can be found in Fig. $2 \mathrm{C}$ with the corresponding photovoltaic characteristics summarized in Table 2.

The optimum D:A ratios were, with efficiencies of $3.92 \%$, $3.33 \%, 2.96 \%$ and $0.75 \%$, found to be $1: 0.8,1: 1.5,1: 1.75$, and 1:2.25 for P3HT:A1, A2, A3, and A4, respectively (as seen in Fig. 3A, C, E, and G). Interestingly, such optimized ratio switches unfavourably towards higher quantity of acceptor as the core becomes less fused and thereby obtains more degrees of freedom. Thinner films were generally found to be favourable, with only P3HT:A1 devices being improved upon increasing the film thickness from around $55 \mathrm{~nm}$ to $70 \mathrm{~nm}$. The average optimized performances were found to be $5.39 \%, 3.37 \%, 3.21 \%$ and $\mathbf{1 . 0 0 \%}$ for devices from P3HT:A1, A2, A3, and A4, respectively, as seen in Fig. 3B, D, F and H. The discovered limitations in film
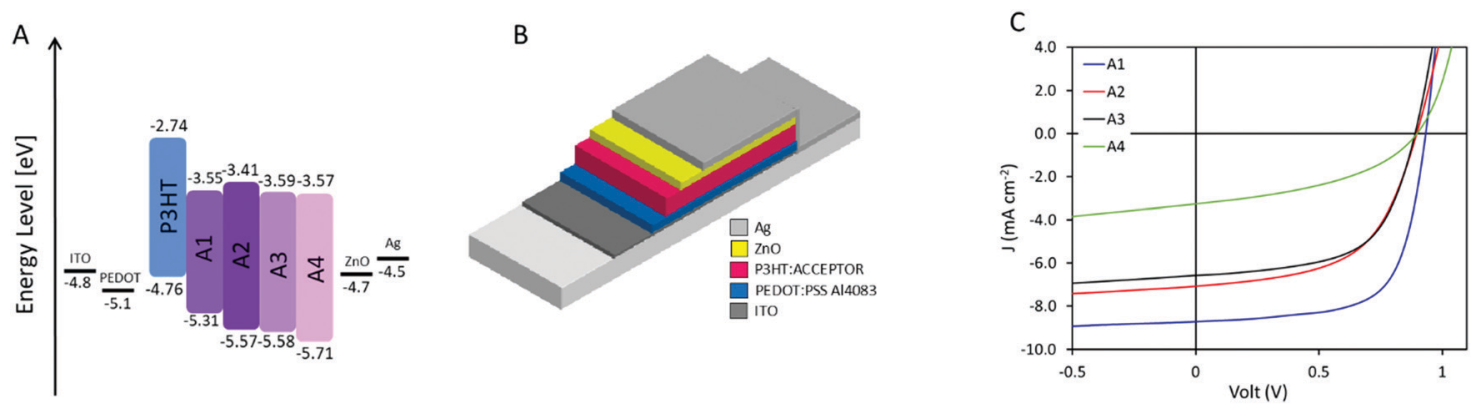

Fig. 2 (A) Energy level diagram for the prepared devices. (B) Schematic presentation of the prepared device structure, being normal architecture with a layer structure of glass/ITO/PEDOT:PSS Al4083/active layer/ZnO/Ag. (C) J-V characteristics of the hero device for the four novel acceptors.
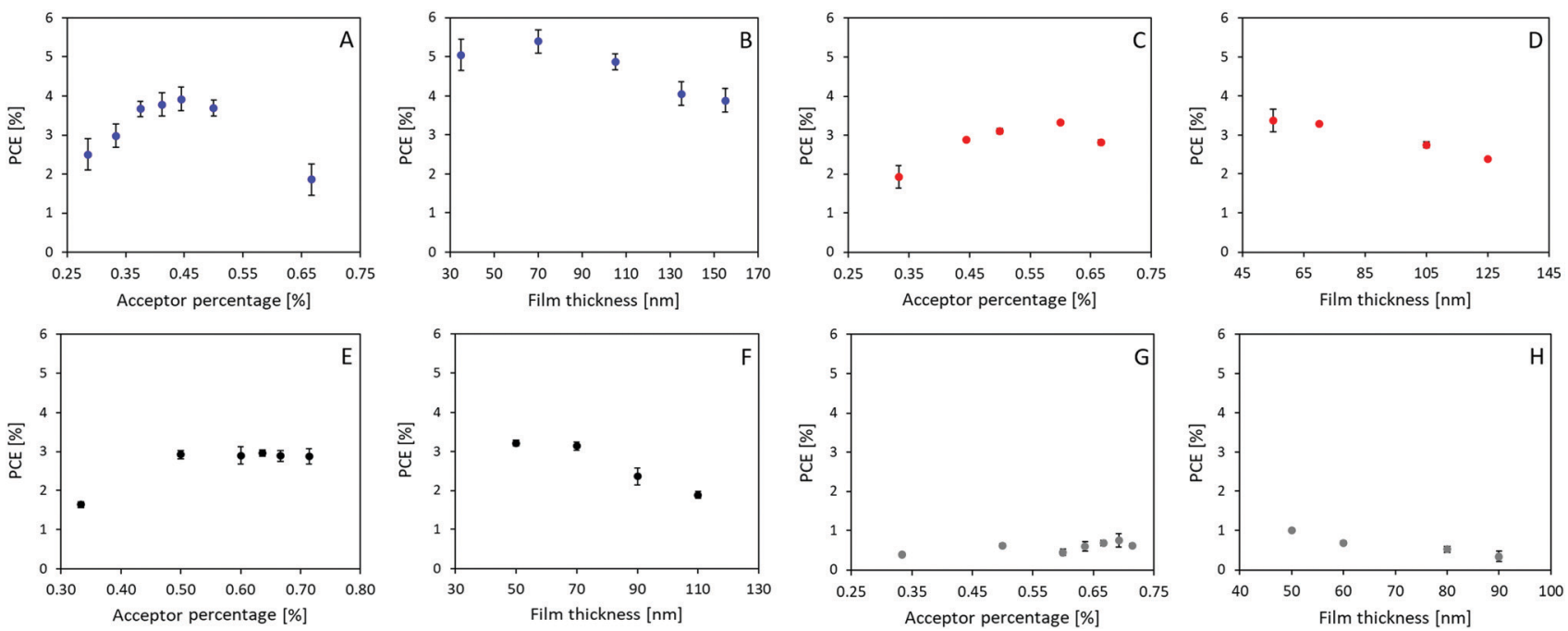

Fig. 3 Device fabrication parameter optimization, namely D:A ratio (acceptor percentage) and active layer thickness (film thickness) presented for P3HT:A1 in A and B, P3HT:A2 in C and D, P3HT:A3 in E and F, and P3HT:A4 in G and H. 
Table 2 Tabular summarization of the photovoltaic characteristics of the optimized devices for each active layer combination, these averages are based on 8 devices with an area of $3.8 \mathrm{~mm}^{2}$

\begin{tabular}{|c|c|c|c|c|c|c|}
\hline & Ratio P3HT: acceptor & Thickness [nm] & $V_{\mathrm{OC}}[\mathrm{V}]$ & $J_{\mathrm{SC}}\left[\mathrm{mA} \mathrm{cm}^{-2}\right]$ & $\mathrm{FF}[\%]$ & PCE $[\%]$ \\
\hline $\mathbf{A 2}$ & $1: 1.50$ & 55 & $0.89 \pm 0.01$ & $6.97 \pm 0.10$ & $53.90 \pm 0.4$ & $3.33 \pm 0.03$ \\
\hline
\end{tabular}

Table 3 Hole and electron mobility determined from SCLC experiments with hole and electron only devices with a structure of glass/ITO/PEDOT:PSS Al4083/active layer/MoOx/Ag and glass/ITO/ZnO/active layer/PFN/Ag, respectively. Charge carrier mobility balance was determined dividing the hole mobility with the electron mobility

\begin{tabular}{lllr}
\hline Acceptor & Hole mobility $\left(\mathrm{cm}^{2}(\mathrm{~V} \mathrm{c})^{-1}\right)$ & Electron mobility $\left(\mathrm{cm}^{2}(\mathrm{~V} \mathrm{c})^{-1}\right)$ & Charge carrier mobility balance \\
\hline A1 & $1.04 \times 10^{-4} \pm 2.19 \times 10^{-5}$ & $5.79 \times 10^{-5} \pm 8.80 \times 10^{-6}$ & 1.8 \\
A2 & $9.85 \times 10^{-5} \pm 2.07 \times 10^{-5}$ & $6.51 \times 10^{-6} \pm 3.42 \times 10^{-6}$ & 15.1 \\
A3 & $7.82 \times 10^{-5} \pm 1.11 \times 10^{-5}$ & $3.40 \times 10^{-6} \pm 7.23 \times 10^{-7}$ & 23.0 \\
A4 & $1.33 \times 10^{-4} \pm 3.43 \times 10^{-5}$ & $4.01 \times 10^{-6} \pm 2.99 \times 10^{-6}$ & 33.2
\end{tabular}

thicknesses could be ascribed to low charge carrier mobilities which inhere were determined through space charge limited current (SCLC) measurements of electron only (glass/ITO/ZnO/ active layer/PFN/Ag) and hole only (glass/ITO/PEDOT:PSS Al4083/ active layer $/ \mathrm{MoO} x / \mathrm{Ag}$ ) devices, the obtained results are summarized in Table 3. As seen all active layers exhibit the values ranging from $7.82 \times 10^{-5} \mathrm{~cm}^{2}(\mathrm{~V} \mathrm{c})^{-1}$ to $1.33 \times 10^{-4} \mathrm{~cm}^{2}(\mathrm{~V} \mathrm{c})^{-1}$ for their reasonably similar hole mobilities, which correlates well with all devices utilizing P3HT as electron donor/hole transport material. The electron mobility is, however, significantly influenced by the choice of acceptor as it varies with more than one order of magnitude with P3HT:A1 reaching an electron mobility of $5.79 \times$ $10^{-5} \mathrm{~cm}^{2}(\mathrm{~V} \mathrm{c})^{-1}$ whereas P3HT:A2, A3, and A4 exhibit their electron mobilities of $\sim 5 \times 10^{-6} \mathrm{~cm}^{2}(\mathrm{~V} \mathrm{c})^{-1}$ clearly explaining the requirement for very thin films. It is evident that P3HT:A2-A4 films have a vastly imbalanced carrier transport with ratios of hole- to electronmobility was found to be between 15 and 33. This suggests the main contributor to the reduced device FF could be imbalanced carrier transport. As for the observed variations in $J_{\mathrm{SC}}$, multiple factors may be contributing such as number of formed excitons, exciton dissociation, and free carrier extraction. The number of excitons would be directly correlated with the light harvesting under controlled film thickness which potentially could explain the differences among devices of P3HT:A1-A3 with varied $J_{\mathrm{sc}}$ of $6.49-8.69 \mathrm{~mA} \mathrm{~cm}{ }^{-2}$ (as P3HT:A1 displays a red-shift in light absorbance and allows for increased film thickness (Fig. 1C and 3)). Exciton dissociation is generally influenced by energy level offsets and the phase-separation between the active layer materials. The energy level offsets are almost identical for the four material combinations presented herein and sufficient to ensure efficient dissociation (LUMO offsets significantly larger than the hypothesized $0.3 \mathrm{eV}$ ). It is therefore more likely that the $J_{\mathrm{SC}}$ for these devices, especially P3HT:A4, is hindered by a non-optimal phase-separation allowing excitons to be recombined prior to dissociation. Free carrier extraction has been discussed above with P3HT:A2-A4 exhibiting a vastly imbalanced carrier mobility.

In order to do a proper quantification of the difference between A1 and O-IDTBR (and other novel materials), the previously published standardized i-FOM was applied for A1 and compared with that for O-IDTBR. ${ }^{11}$ The i-FOM is given by a relationship amongst device efficiency, stability after $200 \mathrm{~h}$ of light exposure, and synthetic complexity (SC) as presented in eqn (1).

$$
\mathrm{i}-\mathrm{FOM}=\frac{\mathrm{PCE} \times \text { Stability }}{\mathrm{SC} \operatorname{index}(M)}
$$

The equation for calculating i-FOM, based on the device PCE, device light stability after $200 \mathrm{~h}$ of light exposure, and the SC index $(M)$.

The SC of A1 was calculated as presented in eqn (2), it was described in literature based on the normalized number of synthetic steps (NSS), the normalized overall reciprocal yield (RY), the normalized number of operation units for purification (NUO), the normalized number of column chromatography for purification (NCC) and the normalized number of hazardous chemicals (NHC). ${ }^{10}$

$$
\begin{aligned}
\mathrm{SC}= & 35 \frac{\mathrm{NSS}}{\mathrm{NSS}_{\max }}+25 \frac{\log (\mathrm{RY})}{\log \left(\mathrm{RY}_{\max }\right)}+15 \frac{\mathrm{NUO}}{\mathrm{NUO}_{\max }} \\
& +15 \frac{\mathrm{NCC}}{\mathrm{NCC}_{\max }}+10 \frac{\mathrm{NHC}^{\mathrm{NHC}_{\max }}}{}
\end{aligned}
$$

The equation for calculating SC with the values for $\mathrm{NSS}_{\max }=22$, $\mathrm{RY}_{\max }=86.9, \mathrm{NUO}_{\max }=39, \mathrm{NCC}_{\max }=13$, and $\mathrm{NHC}_{\text {max }}=44$ were taken from literature. ${ }^{10}$

As presented in Table 4, a SC value of 36.7 was obtained for A1 which is a significant decrease of around 33\% compared with that of 54.9 previously presented for O-IDTBR. This decrease in SC are to be expected as $\mathbf{A 1}$ is a form of simplification on O-IDTBR thereby reducing NSS, whilst the remaining synthetic steps are analogous due to the similarities of the molecules, therefore the increase in NHC from 16 in O-IDTBR to 29 in $\mathbf{A 1}$ caused wonderment. The NHC number presented for $\mathbf{A 1}$ herein was obtained on the basis of the guidelines put up in Po et al. ${ }^{10}$ including purification solvents. The reduced SC value of $\mathbf{A 1}$ becomes even more pronounced compared to that 
Table 4 Top section for calculations of the synthetic complexity (SC) on the basic of normalized number of synthetic steps (NSS), normalized reciprocal yield (RY), normalized number of operation units for purification (NUO), normalized number of column chromatography for purification (NCC) and normalized number of hazardous chemicals (NHC). Bottom section for calculation of the i-FOM based on SCacceptor, $\mathrm{SC}_{\text {donor, }}$ donor : acceptor ratio, $\mathrm{PCE}$, and stability after $200 \mathrm{~h}$ of light exposure

\begin{tabular}{llllll}
\hline Synthetic complexity & Norm_NSS & Norm_RY & Norm_NUO & Norm_NCC & Norm_NHC \\
\hline A1 & 0.32 & 0.28 & 0.41 & 0.38 & 0.66 \\
O-IDTBR & 0.5 & 0.70 & 0.62 & 0.46 & 0.36 \\
\hline i-FOM calculation & Ratio & SC 1 P3HT & SC index (M) [\%] & PCE [\%] & Stability \\
\hline A1 & $1: 0.8$ & 7.7 & 20.6 & 5.39 & $0.98^{a}$ \\
O-IDTBR & $1: 1$ & 7.7 & 31.3 & 6.05 & 0.98 \\
\hline
\end{tabular}

${ }^{a}$ Assumed stability based on P3HT:O-IDTBR.

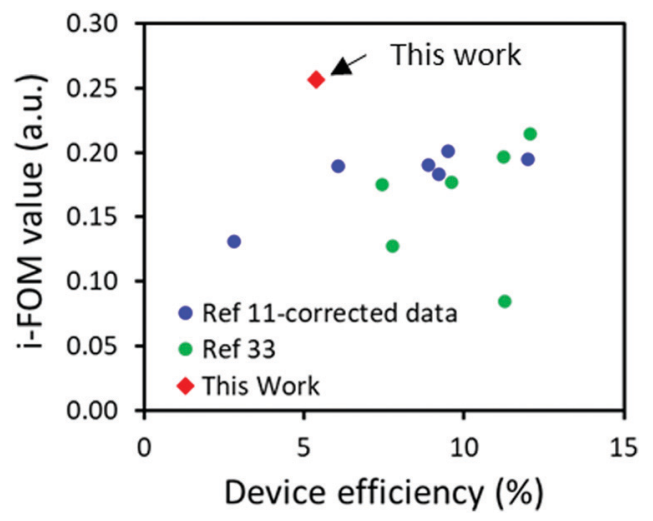

Fig. 4 A comparison of the industrial readiness factors (i-FOM) against previously published ones as a function of device efficiency.

of O-IDTBR when combined with P3HT, as the optimum D:A ratio for $\mathrm{P} 3 \mathrm{HT}: \mathbf{A 1}$ was found to be 1:0.8 compared to 1:1 for P3HT:O-IDTBR. ${ }^{17}$ Since P3HT has a SC score of 7.7 , the combination of P3HT:A1 resulted in an SC index of 20.6 compared to 31.3 for P3HT:O-IDTBR. Such SC index combined with a PCE of 5.39 and an assumed stability of 0.98 (based on the device stability of P3HT:O-IDTBR) results in an i-FOM value of 0.26 which is not only significantly higher than that of 0.19 obtained for P3HT:O-IDTBR, but also the highest i-FOM value ever reported, as seen in Fig. $4 .^{11,35}$ Such a high i-FOM value clearly demonstrates a strong potential of A1 as a stepping stone towards finding an industrial viable donor:acceptor pair for upscaling OSCs. The i-FOM may be increased even further by replacing the fused core with a non-fused core as demonstrated by Chen and coworkers. ${ }^{36}$

\section{Upscaling of P3HT:A1 to large area slot-die coated ITO-free organic solar cells.}

As the active layer material combination of P3HT:A1 with an i-FOM value of 0.26 displays a great potential for being at least a stepping stone towards industrial relevance. Large area ITO-free OSCs with an area of $0.88 \mathrm{~cm}^{2}$ were fabricated and optimized through slot-die coating (except for the $\mathrm{Ag}$ and $\mathrm{Al}$ electrodes which were flexographically printed and thermally evaporated, respectively) with the layer stack of PET/Ag/PEDOT:PSS/P3HT:A1/ZnO/Al in normal geometry as seen in Fig. 5A. In an attempt to make the transfer from small-area spin-coated device to the large-area coated and printed devices less complicated, direct transfer of the D:A ratio of $1: 0.8$ and annealing conditions $140{ }^{\circ} \mathrm{C}$ for $4 \mathrm{~min}$ was adapted. An active layer thickness of $70 \mathrm{~nm}$ was deemed too thin in comparison with the previously reported thinnest one in normal geometry large-area OSCs of $150 \mathrm{~nm}^{8}$. And chloroform, being a very low evaporation temperature solvent, is generally not viewed as an optimal solvent for large-area OSCs fabrication, therefore a solvent mixture of chlorobenzene and chloroform (CB:CF) 90:10 was chosen. ${ }^{37}$ As an initial investigation, the type of additive was determined by preparing devices with $5 \%$ additive of either chloronaphthalene (CN), 1,2,3,4-tetrahydronaphthalene ( $\mathrm{THN}$ ), 4-bromoanisole (BrA), 1,8-diiodooctane (DIO), or ortho-dichlorobenzene (o-DCB). THN, BrA, and $o$-DCB have previously been utilized with P3HT: O-IDTBR with great success. ${ }^{38,39}$ However, from the obtained results herein (Fig. 5B), it can be seen that only DIO or $o$-DCB with PCE of $1.1 \%$ enhances the device efficiency above the CB:CF base solvent mixture. As the material combination have proven to be highly thickness dependent, a series of device with active layer thicknesses ranging from $70-220 \mathrm{~nm}$ were prepared. As illustrated in Fig. 5C, $100 \mathrm{~nm}$ is found to be the optimized active layer thickness with slight decrease in efficiency of approximately $10 \%$ for the a bit thinner one $(70 \mathrm{~nm})$, and with a plateau of $70 \%$ of the optimum for devices with active layer above $150 \mathrm{~nm}$. The underlying reasons for the relatively low device performance were investigated through a thermal annealing test as can be seen in Table S4 in the ESI, $\dagger 140{ }^{\circ} \mathrm{C}$ appears to be too high a temperature as the device efficiency only decreases with extended annealing time. Therefore, the annealing temperature was lowered to $120{ }^{\circ} \mathrm{C}$ for both pre- and post-annealing of the prepared devices for the following experiments. The ink composition was optimized firstly through varying the $o$-DCB concentration, giving a peak efficiency of $3.58 \%$ at $20 \% o$-DCB with low molecular weight P3HT (20 kDa). While high molecular weight P3HT (60 kDa) only allows a hero efficiency of $2.68 \%$ as seen in Fig. 5D. Secondly through variation of chloroform content in the inks as displayed in Fig. 5E, a chloroform concentration of $20 \%$ appears to be optimum with a slight device efficiency increase from $10 \%$ to $20 \%$ before decreasing again at $30 \%$ chloroform in the ink mixture. From Fig. $5 \mathrm{E}$ it is also clearly evident that devices 

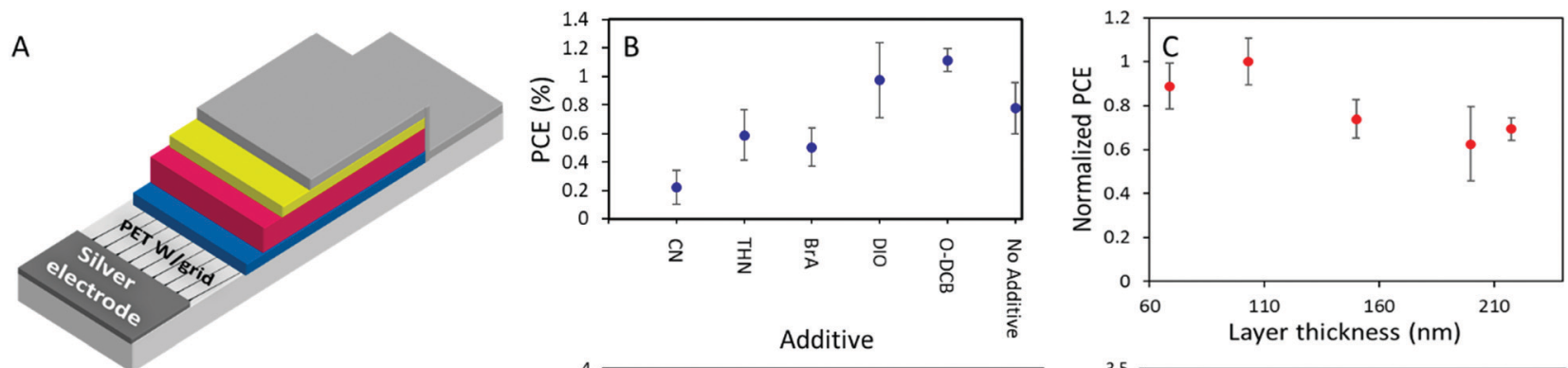

Al (Evaporated)

ZnO (Slot-die coated)

P3HT:A1(Slot-die coated)

PEDOT:PSS PH1000 (Slot-die coated)
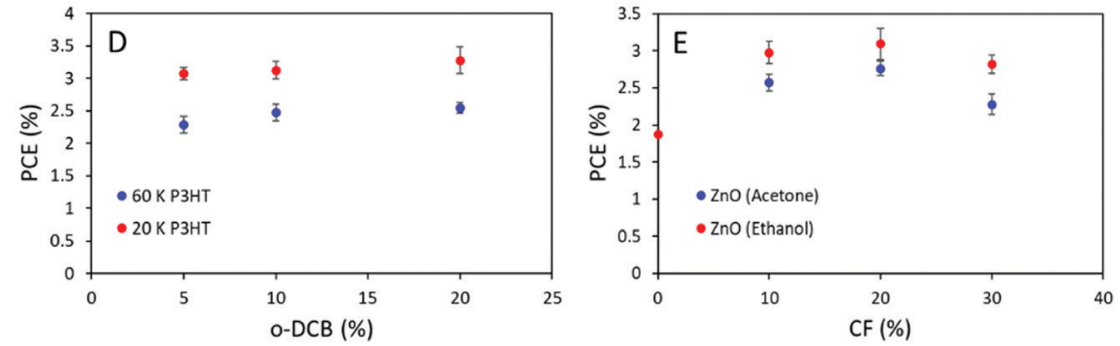

Fig. 5 (A) Schematic presentation of the printed layer-stack for normal geometry flexible OSCs. (B) Device efficiencies as a function of additive with a concentration of $5 \%$ (chloronaphthalene (CN), 1,2,3,4-tetrahydronaphthalene (THN), 4-bromoanisole (BrA), 1,8-diiodooctane (DIO), orthodichlorobenzene (o-DCB)). (C) Normalized device efficiency as a function of active layer thickness. (D) PCE of prepared devices with two different P3HT with $M_{w}$ of $20 \mathrm{kDa}$ and $60 \mathrm{kDa}$, respectively, as a function of $O-D C B$ concentration. (E) Device PCEs as a function of chloroform concentration with $\mathrm{ZnO}$ deposited from either acetone or ethanol.
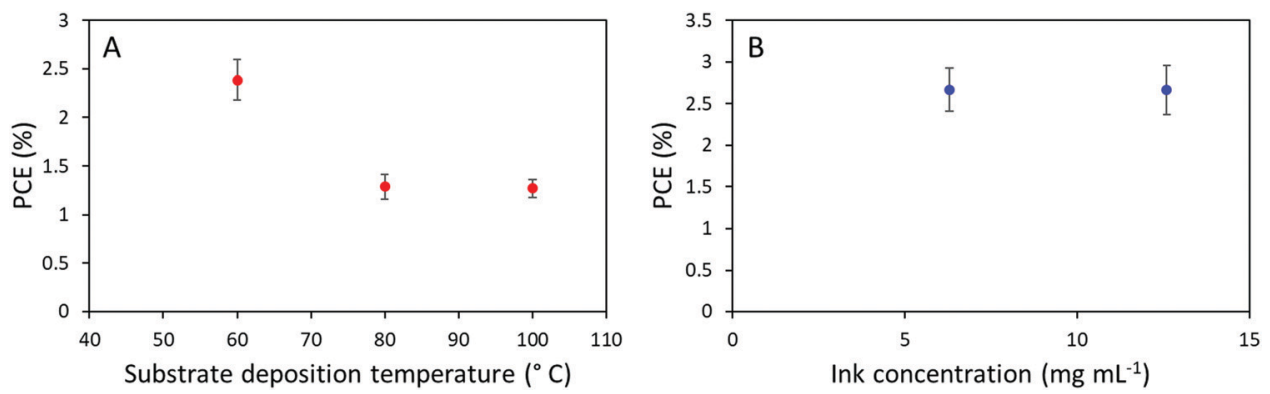

Fig. 6 (A) Device PCE development as a function of substrate deposition temperature. (B) Device efficiency as a function of ink concentration with concentrations of $6.3 \mathrm{mg} \mathrm{mL}^{-1}$ and $12.6 \mathrm{mg} \mathrm{mL}^{-1}$.

with ethanol based $\mathrm{ZnO}$ have superior performance over acetone based ones, likely do to with solubilities as A1 exhibits slight soluble in acetone, leading to a depletion of $\mathbf{A 1}$ in the top part of the active layer and a mixed ETL due to the deposition of acetone based $\mathrm{ZnO}$.

In recent literatures, a multitude of minor deposition variations have proven highly efficient for the sake of enhancing the efficiency of slot-die coated OSCs devices. Initially, Na et al. presented an impressive efficiency optimization from approximately $2.5 \%$ to around $8 \%$ through controlling the substrate and slot-die coating-head temperatures during active layer deposition. ${ }^{40}$ Implementing this approach did, however, not have a positive influence on our prepared devices, as we observed a decrease in device performance from around $2.5 \%$ to $1.2 \%$ with increased substrate temperature from 60 to $80{ }^{\circ} \mathrm{C}$, and a further temperature increase to $100{ }^{\circ} \mathrm{C}$ did not appear to change the performance further, as seen in Fig. 6A. Meng et al. published another approach, in which they significantly decreased the concentration of the ink, illustrating that the obtained morphology can much better emulate spin-coating with a PCE around 9.5\%. ${ }^{41}$ Meanwhile this approach did not result in improved device efficiencies for the system presented herein, as seen in Fig. 6B, no differences were observed in the device efficiency when diluting the active layer ink from $12.6 \mathrm{mg} \mathrm{mL}^{-1}$ to $6.3 \mathrm{mg} \mathrm{mL}^{-1}$.

\section{Conclusion}

In this work, we present four novel small molecule non-fullerene acceptors with four different core-molecules (4,9-dihydro-4,4,9,9tetraoctyl-s-indaceno[1,2-b:5,6- $\left.b^{\prime}\right]$-dithiophene-dicarbaldehyde, 6-(5-formylthiophen-2-yl)-4,4-dioctyl- $4 H$-indeno[1,2- $b]$ thiophene2-carbaldehyde, 5,5,10,10-tetraoctyl-5,10-dihydrothieno[ $\left[2^{\prime}, 3^{\prime}: 3,4\right]$ cyclopenta[1,2-g]thieno[3,2-c] isochromene-2,8-dicarbaldehyde and 5,5'-(2,5-bis((2-ethylhexyl)oxy)-1,4-phenylene)bis(thiophene-2carbaldehyde)) flanked by 3-ethylrhodanine. These molecules were designed as synthetically simplified alternatives to O-IDTBR as acceptors for the best cost-effective donor polymer P3HT. As a 
consequence of the simplified structure of A1-A4, their optical absorbance has a greater overlap with P3HT than previously reported O-IDTBR. The optical band gaps of the acceptor presented herein are between $1.95 \mathrm{eV}$ and $2.05 \mathrm{eV}$, due to their difference in core rigidity allowing $\mathbf{A} 2$ to twist thereby disrupting the molecular packing. A1-A4 exhibited HOMO-levels between $-5.71 \mathrm{eV}$ and $-5.57 \mathrm{eV}$ and LUMO-levels between $-3.41 \mathrm{eV}$ and $-3.59 \mathrm{eV}$, which are suitable for pairing with P3HT. The optimized P3HT:A1-A4 devices yielded efficiencies of 5.39, 3.33, 3.21, and $\mathbf{1 . 0 0 \%}$ for $\mathbf{A 1}, \mathbf{A 2}, \mathbf{A 3}$, and $\mathbf{A 4}$, respectively, with optimum ratios pushed towards higher amount of acceptor with less fused cores. The simplified structure of $\mathbf{A 1}$ combined with the device efficiency of $5.39 \%$ resulted in an i-FOM (industrial readiness measure) value of 0.26 which is more than a $30 \%$ increase over that of O-IDTBR and the highest ever reported i-FOM value. The P3HT:A1 combination was upscaled to large-area ITO-free slot-die coated OSCs, reaching an optimized efficiency up to $3.58 \%$.

\section{Conflicts of interest}

There are no conflicts to declare.

\section{Acknowledgements}

TRA and ATW contributed equally to this work. TRA would like to greatly acknowledge the financial support from National Natural Science Foundation of China (No. 51850410580). QT thanks support from China Scholarship Council (CSC), Grants from Natural Science Foundation of Hunan Province (Grant No. 2015JJ2042, 2018JJ3098) and the Scientific Research Fund of Hunan Provincial Education Department (No. 18A344). Support from Sino-Danish Center for Education and Research is fully acknowledged by DY.

\section{References}

1 S. E. Shaheen, D. S. Ginley and G. E. Jabbour, MRS Bull., 2005, 30, 10-19.

2 C. J. Mulligan, C. Bilen, X. Zhou, W. J. Belcher and P. C. Dastoor, Sol. Energy Mater. Sol. Cells, 2015, 133, 26-31.

3 C. J. Brabec, Sol. Energy Mater. Sol. Cells, 2004, 83, 273-292.

4 B. Azzopardi, C. J. M. Emmott, A. Urbina, F. C. Krebs, J. Mutale and J. Nelson, Energy Environ. Sci., 2011, 4, 3741.

5 F. C. Krebs, Sol. Energy Mater. Sol. Cells, 2009, 93, 394-412.

6 F. C. Krebs, Sol. Energy Mater. Sol. Cells, 2009, 93, 465-475.

7 F. C. Krebs, S. A. Gevorgyan and J. Alstrup, J. Mater. Chem., 2009, 19, 5442-5451.

8 T. R. Andersen, F. Almyahi, N. A. Cooling, D. Elkington, L. Wiggins, A. Fahy, K. Feron, B. Vaughan, M. J. Griffith, A. J. Mozer, C. Sae-kung, G. G. Wallace, W. J. Belcher and P. C. Dastoor, J. Mater. Chem. A, 2016, 4, 15986-15996.

9 T. R. Andersen, N. A. Cooling, F. Almyahi, A. S. Hart, N. C. Nicolaidis, K. Feron, M. Noori, B. Vaughan, M. J. Griffith, W. J. Belcher and P. C. Dastoor, Sol. Energy Mater. Sol. Cells, 2016, 149, 103-109.
10 R. Po, G. Bianchi, C. Carbonera and A. Pellegrino, Macromolecules, 2015, 48, 453-461.

11 N. Li, I. McCulloch and C. J. Brabec, Energy Environ. Sci., 2018, 11, 1355-1361.

12 J. Min, Y. N. Luponosov, C. Cui, B. Kan, H. Chen, X. Wan, Y. Chen, S. A. Ponomarenko, Y. Li and C. J. Brabec, Adv. Energy Mater., 2017, 7, 1700465.

13 J. Yuan, Y. Zhang, L. Zhou, G. Zhang, H.-L. Yip, T.-K. Lau, X. Lu, C. Zhu, H. Peng, P. A. Johnson, M. Leclerc, Y. Cao, J. Ulanski, Y. Li and Y. Zou, Joule, 2019, 3, 1140-1151.

14 Y. Lin, J. Wang, Z.-G. Zhang, H. Bai, Y. Li, D. Zhu and X. Zhan, Adv. Mater., 2015, 27, 1170-1174.

15 S. Li, L. Ye, W. Zhao, X. Liu, J. Zhu, H. Ade and J. Hou, Adv. Mater., 2017, 29, 1704051.

16 B. Xiao, A. Tang, J. Yang, Z. Wei and E. Zhou, ACS Macro Lett., 2017, 6, 410-414.

17 S. Holliday, R. S. Ashraf, A. Wadsworth, D. Baran, S. A. Yousaf, C. B. Nielsen, C.-H. Tan, S. D. Dimitrov, Z. Shang, N. Gasparini, M. Alamoudi, F. Laquai, C. J. Brabec, A. Salleo, J. R. Durrant and I. McCulloch, Nat. Commun., 2016, 7, 11585.

18 S. Li, W. Liu, M. Shi, J. Mai, T.-K. Lau, J. Wan, X. Lu, C.-Z. Li and H. Chen, Energy Environ. Sci., 2016, 9, 604-610.

19 W. Liu, S. Li, J. Huang, S. Yang, J. Chen, L. Zuo, M. Shi, X. Zhan, C.-Z. Li and H. Chen, Adv. Mater., 2016, 28, 9729-9734.

20 J. I. Khan, R. S. Ashraf, M. A. Alamoudi, M. N. Nabi, H. N. Mohammed, A. Wadsworth, Y. Firdaus, W. Zhang, T. D. Anthopoulos, I. McCulloch and F. Laquai, Sol. RRL, 2019, 3, 1900023.

21 A. Wadsworth, Z. Hamid, M. Bidwell, R. S. Ashraf, J. I. Khan, D. H. Anjum, C. Cendra, J. Yan, E. Rezasoltani, A. A. Y. Guilbert, M. Azzouzi, N. Gasparini, J. H. Bannock, D. Baran, H. Wu, J. C. de Mello, C. J. Brabec, A. Salleo, J. Nelson, F. Laquai and I. McCulloch, Adv. Energy Mater., 2018, 8, 1801001.

22 N. Wang, W. Yang, S. Li, M. Shi, T.-K. Lau, X. Lu, R. Shikler, C.-Z. Li and H. Chen, Chin. Chem. Lett., 2019, 30, 1277-1281.

23 X. Xu, G. Zhang, L. Yu, R. Li and Q. Peng, Adv. Mater., 2019, 31, 1906045.

24 S. Li, L. Zhan, F. Liu, J. Ren, M. Shi, C.-Z. Li, T. P. Russell and H. Chen, Adv. Mater., 2018, 30, 1705208.

25 Q. Tao and D. Yu, to be published.

26 W. Zhang, J. Smith, S. E. Watkins, R. Gysel, M. McGehee, A. Salleo, J. Kirkpatrick, S. Ashraf, T. Anthopoulos, M. Heeney and I. McCulloch, J. Am. Chem. Soc., 2010, 132, 11437-11439.

27 H. Chen, A. Wadsworth, C. Ma, A. Nanni, W. Zhang, M. Nikolka, A. M. T. Luci, L. M. A. Perdigão, K. J. Thorley, C. Cendra, B. Larson, G. Rumbles, T. D. Anthopoulos, A. Salleo, G. Costantini, H. Sirringhaus and I. McCulloch, J. Am. Chem. Soc., 2019, 141, 18806-18813.

28 L. Cai, T. Moehl, S.-J. Moon, J.-D. Decoppet, R. HumphryBaker, Z. Xue, L. Bin, S. M. Zakeeruddin and M. Grätzel, Org. Lett., 2014, 16, 106-109.

29 G. Zeng, J. Ouyang, Y. Xin, X. Zhao and X. Yang, J. Mater. Chem. C, 2018, 6, 13211-13217.

30 S. Li, L. Zhan, T.-K. Lau, Z.-P. Yu, W. Yang, T. R. Andersen, Z. Fu, C.-Z. Li, X. Lu, M. Shi and H. Chen, Small Methods, 2019, 3, 1900531. 
31 S. Li, L. Zhan, C. Sun, H. Zhu, G. Zhou, W. Yang, M. Shi, C.Z. Li, J. Hou, Y. Li and H. Chen, J. Am. Chem. Soc., 2019, 141, 3073-3082.

32 S. Li, L. Zhan, W. Zhao, S. Zhang, B. Ali, Z. Fu, T.-K. Lau, X. Lu, M. Shi, C.-Z. Li, J. Hou and H. Chen, J. Mater. Chem. A, 2018, 6, 12132-12141.

33 N. Wang, L. Zhan, S. Li, M. Shi, T.-K. Lau, X. Lu, R. Shikler, C.-Z. Li and H. Chen, Mater. Chem. Front., 2018, 2, 2006-2012.

34 L. Zhan, S. Li, T.-K. Lau, Y. Cui, X. Lu, M. Shi, C.-Z. Li, H. Li, J. Hou and H. Chen, Energy Environ. Sci., 2020, 13, 635-645.

35 X. Du, T. Heumueller, W. Gruber, A. Classen, T. Unruh, N. Li and C. J. Brabec, Joule, 2019, 3, 215-226.

36 Z.-P. Yu, Z.-X. Liu, F.-X. Chen, R. Qin, T.-K. Lau, J.-L. Yin, X. Kong, X. Lu, M. Shi, C.-Z. Li and H. Chen, Nat. Commun., 2019, 10, 1-9.
37 T. R. Andersen, H. F. Dam, B. Andreasen, M. Hösel, M. V. Madsen, S. A. Gevorgyan, R. R. Søndergaard, M. Jørgensen and F. C. Krebs, Sol. Energy Mater. Sol. Cells, 2014, 120, 735-743.

38 S. Strohm, F. Machui, S. Langner, P. Kubis, N. Gasparini, M. Salvador, I. McCulloch, H.-J. Egelhaaf and C. J. Brabec, Energy Environ. Sci., 2018, 11, 2225-2234.

39 E. Pascual-San-José, X. Rodríguez-Martínez, R. Adel-Abdelaleim, M. Stella, E. Martínez-Ferrero and M. Campoy-Quiles, J. Mater. Chem. A, 2019, 7, 20369-20382.

40 S.-I. Na, Y.-H. Seo, Y.-C. Nah, S.-S. Kim, H. Heo, J.-E. Kim, N. Rolston, R. H. Dauskardt, M. Gao, Y. Lee and D. Vak, Adv. Funct. Mater., 2019, 29, 1805825.

41 X. Meng, L. Zhang, Y. Xie, X. Hu, Z. Xing, Z. Huang, C. Liu, L. Tan, W. Zhou, Y. Sun, W. Ma and Y. Chen, Adv. Mater., 2019, 31, 1903649. 